Dermatologische Zeitschrift. 1930;59:I-IV

\title{
Contents, Vol. 59, 1930
}

Inhaltsverzeichnis.

Originalarbeiten.

\& $\quad$ Suite

Beck, S. C, Zur Kenntnis der Dermatofibrosarkonm protuborans.

(Kuznitzky-Grabisch, Darier-Ferrand, E. Hoffmannseher Typus des

Hautsarkoms) 1

Beirach, I. S., R. I. Langermann und I. W. Chorosch, Die Iono-

pborese bei Komplikationen der männlichen Gonorrhoe 208

Bergel, S., Neue Ergebnisse der experimentellen Syphilisforschung fur

die Biologie der menscblicben Lues 357

Bernstein, F., Atypisebe Erythemeruption bei einem Karzinomkranken

mit Lokalisation an den Extremitäten, der Mundschleimbaut und den

Bestrahlungsfeldern am Stamm 196

Bernstein, F., Über die Spalthefebefunde beim seborrhoisclieii Ekzem 385 Brill, E., Atypische, multilokulärc. nicht hereditäre Keratodermie auf

kongenitaler Grundlage $\quad 77$

Chorosch, I. W., siehe Beirach, I. S.

Geiger, Richard, Über seltene gonorrhoische Komplikationen . . . 223

Grimm, 0., Die Trichomonas vaginalis Urethritis beim Manne .... 314

Grimm, 0., siehe Ivulisch, R.

Grundmann, H., siehe Nathan, E.

Haack, Karl, und Gerhard Kreyenberg, Vergleichende kapillar-

mikroskopische Untersucliungen an früher sogenannten ,puellae

publicae" und dem Durchschnittsmaterial einer dermatologisch-vene-

rologischen Abteilung 95

Hoffmann, Erich, Über die nach innen gerichtete Schutz- und Heil-

kraft der Haut (Esophylaxie) 155

- 1st Syphilis heilbar?279

Karrenberg, C. L., Zur Beliandlung der Acrodermatitis atropbicans . 166

Krey enberg, Gerhard, siehe Haack, Karl.

Kulisch, Li., und 0. Grimm, Die Trichomonas vaginalis Kolpitis . . 291

Langermann, R. I., siehe Beirach, I. S.

Makarjin, A. A., siehe Pawlow, S. T.

Napp, Victor, Über einen Fall von Epide $\pi$ nolysis bullosa dystrophi-

cans liereditaria tarcla $1 \mathrm{U} 3$

Nathan, E., und H. Grundmann, Cliemisclie Analyse der Hautüber-

(îiiiptindlichkeit nach experimenteller Sensibilisierung mit Salvarsan 367 N cub erg, Hanns, Ein

Beitrag zu der Lehre von der Komplement-

bindungsreaktion bei Gonorrhoe 297 
Nitta, J., Über die Wirkung ciniger ätherischer Ole auf Gonokokken im Reagenzglase und im Peritoneum der weißen Maus

Pawlow, S. T., und A. A. Makarjin, Zur Frage von tumorartigen

Formeu des Lupus erythematodes 111.

Petschnikow, J. D, und A. D. Zelischtschewa, Die Bedeutung

der Bordet-Gengou-Reaktion in der Klinik der Gonorrhoe 303

IV Inlialtsverzcichnis.

«eit,e

Sprafke, $1 \cdot$ I., Balanitis xerotica obliterans Stühmer (Hierzu Tafel I) . .

V oh wink el, K. II., Über die Salvarsan-Sublimat-Mischspritze. Zehn-

jährige Erfahrungen der Tübinger Universitätshautklinik 16

Wucherpfennig, V., Zur Behandlung der Darierschen Erkrankung 374

Zelischtschewa, A. D., siehe Petschnikow, J. D.

Gesellschaftsberichte.

Berliner Dermatologische Gese \schaft. Sitzung vom 11.111.1980 ....

Sitzung vom IB. V. $1930 \quad$ : . 229

Sitzung vom 17. V. $1930 \quad 395$

Sitzung vom 17. VI. $1930 \quad 403$

Köluer Dermatologische Gesellschaft. Sitzung vom 14. IV7. 1930 ....

- $\quad$ Sitzung vom 16. V. $1930 \quad 241$

Deutsche Gesellschaft zur Bekämpfung der Geschlcchtskrankheiten.

28. Jahresversammlung am 30. und 31, V. 1930 in Dresden 136

8. internationaler Dermatologenkongreß in Kopenhagen 355

North of England Dermatological Society. Sitzung vom 7. II. 1930 . . . 250

- $\quad$ Sitzung vom 14. III. $1930 \quad 414$

Royal Society of Medicine. Section of Dermatology. Sitzungen vom

21. XI. und 19. XII. 1929, 16. 1. 1930 243

- $\quad$ Sitzungen vom 20. II. und 20. III. $1930 \quad 410$

Société Française de Dermatologie et de Syphiligraphie. Sitzung vom

14. III. $1929 \quad 320$

Periodische Literatur. Zusammengestellt von Dr. Arthur Alexander-

Charlottenburg $\quad 35,187,251,825,416$

Buchbesprechungen 71, 150, 275, 352, 451

20 Jahre Salvarsan $\quad 854$

Perso $\pi$ alien und Tagesnachrichten $\quad 76,154,278,856,454$

S. C. Beck $\dagger \quad 454$

Prof. Dr. Johann Fabry $\uparrow$

Dr. Carl Gutmann $†$

Carl Leiner $\dagger$ 153

Gedenkfeier für Fritz Schaudinn

Sachregister Namenregister 456465 